\title{
Using a Mixture of N-Best Lists from Multiple MT Systems in Rank-Sum-Based Confidence Measure for MT Outputs *
}

\author{
Yasuhiro Akiba ${ }^{\dagger \dagger}$, Eiichiro Sumita ${ }^{\dagger}$, Hiromi Nakaiwa ${ }^{\dagger}$, \\ Seiichi Yamamoto ${ }^{\dagger}$, and Hiroshi G. Okuno ${ }^{\ddagger}$ \\ $\dagger$ ATR Spoken Language Translation Research Laboratories \\ 2-2-2 Hikaridai, Keihana Science City, Kyoto 619-0288, Japan \\ $\ddagger$ Graduate School of Informatics, Kyoto University \\ Yoshida-Honmachi, Sakyo-ku, Kyoto 606-8501, Japan \\ \{yasuhiro.akiba, eiichiro.sumita, hiromi.nakaiwa seiichi.yamamoto\}@atr.jp, and okuno@i.kyoto-u.ac.jp
}

\begin{abstract}
This paper addressees the problem of eliminating unsatisfactory outputs from machine translation (MT) systems. The authors intend to eliminate unsatisfactory MT outputs by using confidence measures. Confidence measures for MT outputs include the rank-sum-based confidence measure (RSCM) for statistical machine translation (SMT) systems. RSCM can be applied to non-SMT systems but does not always work well on them. This paper proposes an alternative RSCM that adopts a mixture of the $\mathrm{N}$-best lists from multiple MT systems instead of a single-system's N-best list in the existing RSCM. In most cases, the proposed RSCM proved to work better than the existing RSCM on two non-SMT systems and to work as well as the existing RSCM on an SMT system.
\end{abstract}

\section{Introduction}

This paper addresses the challenging problem of eliminating unsatisfactory outputs from machine translation (MT) systems, which are subsystems of a speech-to-speech machine translation (S2SMT) system. The permissible range of translation quality by MT/S2SMT systems depends on the user. Some users permit only perfect translations, while other users permit even translations with flawed grammar. Unsatisfactory MT outputs are those whose translation quality is worse than the level the user can permit.

In this paper, the authors intend to eliminate unsatisfactory outputs by using confidence measures for MT outputs. The confidence measures ${ }^{1}$ indicate how perfect/satisfactory the MT outputs are. In the

\footnotetext{
* This research was supported in part by the Ministry of Public Management, Home Affairs, Posts and Telecommunications, Japan.

${ }^{1}$ These confidence measures are a kind of automatic evaluator such as mWER (Niessen et al., 2000) and BLEU (Papineni et al., 2001). While mWER and BLEU cannot be used online, these confidence measures can. This is because the former are based on reference translations, while the latter is not.
}

discipline of MT, confidence measures for MT outputs have rarely been investigated.

The few existing confidence measures include the rank-sum-based confidence measure (RSCM) for statistical machine translation (SMT) systems, $C_{\text {rank }}$ in (Ueffing et al., 2003). The basic idea of this confidence measure is to roughly calculate the word posterior probability by using ranks of MT outputs in an N-best list from an SMT system. In the discipline of non-parametric statistical test, ranks of numerical values are commonly used instead of the numerical values themselves for statistical tests. In the case of the existing RSCM, the ranks of probabilities of MT outputs in the N-best list were used instead of the probabilities of the outputs themselves. The existing RSCM scores each word in an MT output by summing the complemented ranks of candidates in the N-best list that contain the same word in a Levenshtein-aligned position (Levenshtein, 1966). When the confidence values of all words in the MT output are larger than a fixed threshold, the MT output is judged as correct/perfect. Otherwise, the output is judged as incorrect/imperfect.

The existing RSCM does not always work well

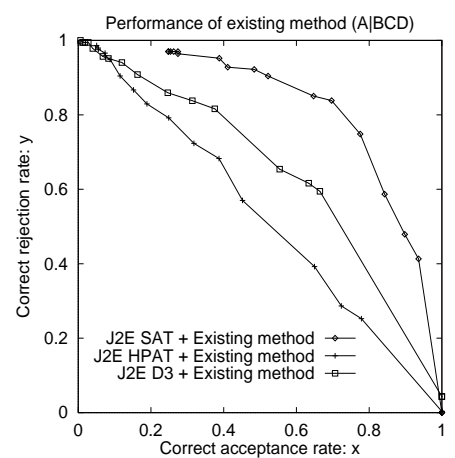

Figure 1: Performance of the existing RSCM on three different types of Japanese-to-English (J2E) MT systems: $\mathrm{D}^{3}$, HPAT, and SAT. The existing RSCM tried to accept perfect MT outputs (grade A in Section 4) and to reject imperfect MT outputs (grades B, C, and D in Section 4). 
on types of MT systems other than SMT systems. Figure 1 shows the differences among the performances, indicated by the Receiver Operating Characteristics (ROC) curve (Section 4.1), of the existing RSCM on each of three MT systems (Section 4.2.1): $\mathrm{D}^{3}$, HPAT, and SAT (Doi and Sumita, 2003; Imamura et al., 2003; Watanabe et al., 2003). Only SAT is an SMT system; the others are not. The ideal ROC curve is a square $(0,1),(1,1),(1,0)$; thus, the closer the curve is to a square, the better the performance of the RSCM is. The performances of the existing RSCM on the non-SMT systems, $\mathrm{D}^{3}$ and HPAT, are much worse than that on the SMT system, SAT.

The performance of the existing RSCM depends on the goodness/density of MT outputs in the Nbest list from the system. However, the system's $\mathrm{N}$-best list does not always give a good approximation of the total summation of the probability of all candidate translations given the source sentence/utterance. The N-best list is expected to approximate the total summation as closely as possible.

This paper proposes a method that eliminates unsatisfactory top output by using an alternative RSCM based on a mixture of N-best lists from multiple MT systems (Figure 2). The elimination system is intended to be used in the selector architecture, as in (Akiba et al., 2002). The total translation quality of the selector architecture proved to be better than the translation quality of each element MT system. The final output from the selection system is the best among the satisfactory top ${ }^{2}$ outputs from the elimination system. In the case of Figure 2, the selection system can receive zero to three top MT outputs. When the selection system receive fewer than two top MT outputs, the selection system merely passes a null output or the one top MT output.

The proposed RSCM differs from the existing RSCM in its N-best list. The proposed RSCM re-

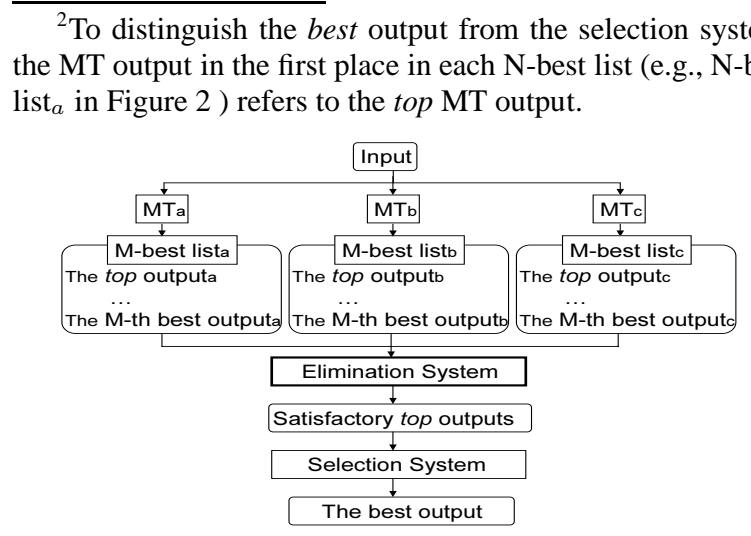

Figure 2: Image of our eliminator ceives an M-best list from each element MT system. Next, it sorts the mixture of the MT outputs in all M-best lists in the order of the average product (Section 3.2) of the scores of a language model and a translation model (Akiba et al., 2002). This sorted mixture is used instead of the system's N-best list in the existing RSCM.

To experimentally evaluate the proposed RSCM, the authors applied the proposed RSCM and the existing RSCM to a test set of the Basic Travel Expression Corpus (Takezawa et al., 2002). The proposed RSCM proved to work better than the existing RSCM on the non-SMT systems and to work as well as the existing RSCM on the SMT system.

The next section outlines the existing RSCM. Section 3 proposes our RSCM. Experimental results are shown and discussed in Section 4. Finally, our conclusions are presented in Section 5.

\section{The Existing RSCM}

The existing confidence measures include the ranksum-based confidence measure (RSCM) for SMT systems (Ueffing et al., 2003). The basic idea of this RSCM is to roughly calculate the word posterior probability by using ranks of MT outputs in the $\mathrm{N}$-best list of an SMT system. That is, the ranks of probabilities of MT outputs in the N-best list were used instead of the probabilities of the outputs themselves, as in the non-parametric statistical test.

Hereafter, $\hat{e}_{1}^{I}$ and $w_{1}^{I_{n}}$ denote the top output ${ }^{2}$ and the $n$-th best output in the N-best list, respectively. $\hat{e}_{i}$ denotes the $i$-th word in the top MT output $\hat{e}_{1}^{I} . L_{i}\left(\hat{e}_{1}^{I}, w_{1}^{I_{n}}\right)$ denote the Levenshtein alignment ${ }^{3}$ (Levenshtein, 1966) of $\hat{e}_{i}$ on the $n$-th best output $w_{1}^{I_{n}}$ according to the top output $\hat{e}_{1}^{I}$. The existing RSCM of the word $\hat{e}_{i}$ is the sum of the ranks of MT outputs in an N-best list containing the word $\hat{e}_{i}$ in a position that is aligned to $i$ in the Levenshtein alignment, which is normalized by the total rank sum:

$$
C_{\text {rank }}\left(\hat{e}_{i}\right)=\frac{\sum_{n=1}^{N}(N-n) \cdot \delta\left(\hat{e}_{i}, L_{i}\left(\hat{e}_{1}^{I}, w_{1}^{I_{n}}\right)\right)}{N(N+1) / 2},
$$

where $\delta(\cdot, \cdot)$ is the Kronecker function, that is, if words/morphemes $x$ and $y$ are the same, $\delta(x, y)=$ 1 ; otherwise, $\delta(x, y)=0$. Thus, only in the case where $\hat{e}_{i}$ and $L_{i}\left(\hat{e}_{1}^{I}, w_{1}^{I_{n}}\right)$ are the same, the rank of the MT output $w_{1}^{I_{n}}, N-n$, is summed. In the calculation of $C_{\text {rank }}, N-n$ is summed instead of the rank $n$ because ranks near the top of the N-best list contribute more to the score $C_{\text {rank }}$.

\footnotetext{
${ }^{3}$ This is the word on the $n$-th best output $w_{1}^{I_{n}}$, aligned with the $i$-th word $\hat{e}_{i}$, in the calculation of edit distance from the top MT output $\hat{e}_{1}^{I}$ to the $n$-th best output $w_{1}^{I_{n}}$.
} 
In this paper, the calculation of $C_{\text {rank }}$ is slightly modified to sum $N-n+1$ so that the total summation is equal to $N(N+1) / 2$. Moreover, when there are MT outputs that have the same score, such MT outputs are assigned the average rank as in the discipline of non-parametric statistical test.

As shown in Section 1, the existing RSCM does not always work well on types of MT systems other than SMT systems. This is because the system's $\mathrm{N}$-best list does not always give a good approximation of the total summation of the probability of all candidate translations given the source sentence/utterance. The N-best list is expected to approximate the total summation as closely as possible.

\section{Proposed Method}

In this section, the authors propose a method that eliminates unsatisfactory top output by using an alternative RSCM based on a mixture of N-best lists from multiple MT systems. The judgment that the top output is satisfactory is based on the same threshold comparison as the judgment that the top output is perfect, as mentioned in Section 1. The elimination system and the alternative RSCM are explained in Sections 3.1 and 3.2, respectively.

\subsection{Elimination system}

This section proposes a method that eliminates unsatisfactory top outputs by using an alternative RSCM based on a mixture of N-best lists from multiple MT systems (Figure 3). This elimination system is intended to be used in the selector architecture (Figure 2). The elimination system receives an M-best list from each element MT system and outputs only top ${ }^{2}$ outputs whose translation quality is better than or as good as that which the user can permit. In the case of Figure 3, the number of MT systems is three; thus, the elimination system can output zero to three top MT outputs, which depends on the number of the eliminated top outputs.

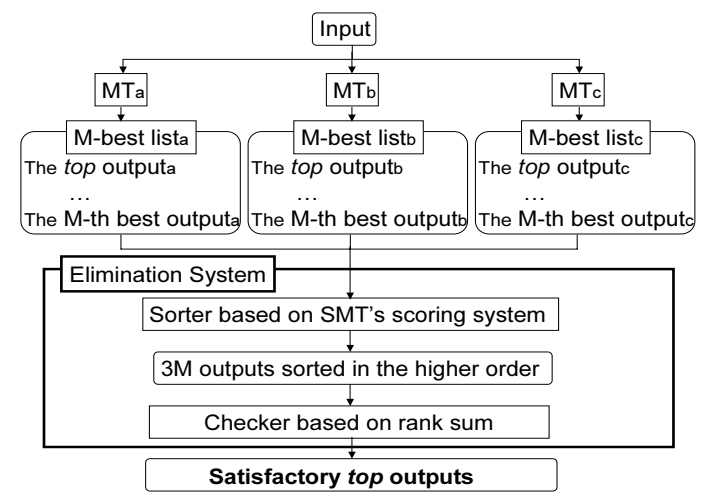

Figure 3: Proposed RSCM
The proposed elimination system judges whether a top output is satisfactory by using a threshold comparison, as in (Ueffing et al., 2003). When the confidence values of all words in the top output, which are calculated by using the alternative RSCM explained in Section 3.2, are larger than a fixed threshold, the top output is judged as satisfactory. Otherwise, the top output is judged as unsatisfactory. The threshold was optimized on a development corpus.

\subsection{The proposed RSCM}

The proposed RSCM is an extension of the existing RSCM outlined in Section 2. The proposed RSCM differs from the existing RSCM in the adopted Nbest list (Figure 3). The proposed RSCM receives an M-best list from each element MT system. Next the proposed RSCM sorts the mixture of all the MT outputs in the order of the average product of the scores of a language model and a translation model (Akiba et al., 2002). This sorted mixture is alternatively used instead of the system's N-best list in the existing RSCM. That is, the proposed RSCM checks whether it accepts/rejects each top MT output in the original M-best lists by using the sorted mixture; on the other hand, the existing RSCM checks whether it accepts/rejects the top MT output in the system's N-best list by using the system's N-best.

For scoring MT outputs, the proposed RSCM uses a score based on a translation model called IBM4 (Brown et al., 1993) (TM-score) and a score based on a language model for the translation target language (LM-score). As Akiba et al. (2002) reported, the products of TM-scores and LM-scores are statistical variables. Even in the case where the translation model (TM) and the language model for the translation target language (LM) are trained on a sub-corpus of the same size, changing the training corpus also changes the TM-score, the LM-score, and their product. Each pair of TM-score and LMscore differently order the MT outputs.

For robust scoring, the authors adopt the multiple scoring technique presented in (Akiba et al., 2002). The multiple scoring technique prepares

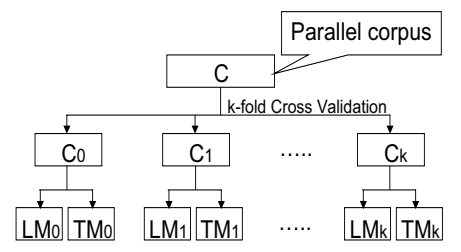

Figure 4: Method for training multiple pairs of Language Models (LMs) and Translation Models (TMs) (Akiba et al., 2002). 
multiple subsets of the full parallel corpus according to k-fold cross validation (Mitchell, 1997) and trains both TM and LM on each subset. Each MT output is scored in k ways. For example, the full parallel corpus $C$ is divided into three subsets $V_{i}(i=0,1,2)$. For each $i$, the proposed method trains a translation model $T M_{i}$ on $C_{i}\left(=C-V_{i}\right)$ and a language model $L M_{i}$ on the target-language part of $C_{i}$ (Figure 4). MT outputs in the mixture are sorted by using the average of the product scores by $T M_{i}$ and $L M_{i}$ for each $i$. In (Akiba et al., 2002), this multiple scoring technique was shown to select the best translation better than a single scoring technique that uses TM and LM trained from a full corpus.

\section{Experimental Comparison}

The authors conducted an experimental comparison between the proposed RSCM and the existing RSCM in the framework of the elimination system. The task of both RSCMs was to judge whether each top $^{2}$ MT output from an MT system is satisfactory, that is, whether the translation quality of the top MT output is better than or as good as that which the user can permit.

In this experiment, the translation quality of MT outputs was assigned one of four grades: A, B, C, or D as follows: (A) Perfect: no problems in either information or grammar; (B) Fair: easy-tounderstand, with either some unimportant information missing or flawed grammar; (C) Acceptable: broken, but understandable with effort; (D) Nonsense: important information has been translated incorrectly. This evaluation standard was introduced by Sumita et al. (1999) to evaluate S2SMT systems. In advance, each top MT output was evaluated by nine native speakers of the target language, who were also familiar with the source language, and then assigned the median grade of the nine grades.

To conduct a fair comparison, the number of MT outputs in the system's $\mathrm{N}$-best list and the number of MT outputs in the mixture are expected to be the same. Thus, the authors used either a threebest list from each of three MT systems or a fivebest list from each of two non-SMT MT systems for the proposed RSCM and a ten-best list for the existing RSCM. Naturally, this setting ${ }^{4}$ is not disadvantageous for the existing RSCM.

\footnotetext{
${ }^{4}$ In the future, we will conduct a large-scale experiment to investigate how both RSCMs work while increasing the size of the system's N-best list and the mixture of M-best lists.
}

Table 1: Confusion matrix

\begin{tabular}{|l|c|c|c|}
\hline & Accept & Reject & Subtotal \\
\hline Satisfactory & $V_{s, a}$ & $V_{s, r}$ & $V_{s}\left(=V_{s, a}+V_{s, r}\right)$ \\
\hline Unsatisfactory & $V_{u, a}$ & $V_{u, r}$ & $V_{u}\left(=V_{u, a}+V_{u, r}\right)$ \\
\hline
\end{tabular}

\subsection{Evaluation metrics}

The performances of both RSCMs were evaluated by using three different metrics: ROC Curve, Hmean, and Accuracy. For each MT system, these metrics were separately calculated by using a confusion matrix (Table 1). For example, for J2E $\mathrm{D}^{3}$ (Section 4.2.1), the proposed RSCM checked each top MT output from J2E $\mathrm{D}^{3}$ by using the input mixture of three-best lists from the three J2E MT systems (Section 4.2.1); on the other hand, the existing RSCM checked each top MT output from J2E $\mathrm{D}^{3}$ by using the input ten-best list from $\mathrm{J} 2 \mathrm{E} \mathrm{D}^{3}$. For $\mathrm{J} 2 \mathrm{E} \mathrm{D}^{3}$, the results were counted up into the confusion matrix of each RSCM, and the metrics were calculated as follows:

ROC Curve plots the correct acceptance rate versus the correct rejection rate for different values of the threshold. Correct acceptance rate (CAR) is defined as the number of satisfactory outputs that have been accepted, divided by the total number of satisfactory outputs, that is, $V_{s, a} / V_{s}$ (Table 1). Correct rejection rate (CRR) is defined as the number of unsatisfactory outputs that have been rejected, divided by the total number of unsatisfactory outputs, that is, $V_{u, r} / V_{u}$ (Table 1 ).

H-mean is defined as a harmonic mean ${ }^{5}$ of the CAR and the CRR (Table 1), $2 * C A R *$ $C R R /(C A R+C R R)$.

Accuracy is defined as a weighted mean ${ }^{6}$ of the CAR and the CRR (Table 1), $\left(V_{s} * C A R+V_{u} *\right.$ $C R R) /\left(V_{s}+V_{u}\right)=\left(V_{s, a}+V_{u, r}\right) /\left(V_{s}+V_{u}\right)$.

For each performance of H-mean and Accuracy, 10 -fold cross validation was conducted. The threshold was fixed such that the performance was maximized on each non-held-out subset, and the performance was calculated on the corresponding held-out subset. To statistically test the differences in performance (H-mean or Accuracy) between the confidence measures, the authors conducted a pairwise ttest (Mitchell, 1997), which was based on the results of 10 -fold cross validation. When the difference in performance meets the following condition, the difference is statistically different at a confidence level

\footnotetext{
${ }^{5}$ This harmonic mean is used for summarizing two measures, each of which has a trade-off relationship with each other. For example, F-measure is the harmonic mean of precision and recall, which is well used in the discipline of Information Retrieval.

${ }^{6}$ This weighted mean is used for evaluating classification tasks in the discipline of Machine Learning.
} 


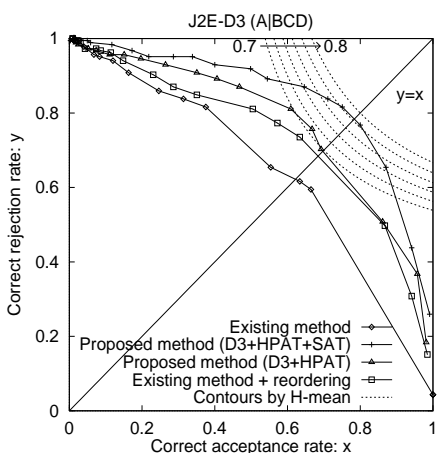

Figure 5: ROC Curves of both RSCMs for J2E-D 3

Table 2: Performance of MT systems: Each number in the $\mathrm{AB}$ row indicates the ratio of A-or-B-graded translation by each MT system. Each number in the other rows similarly indicates corresponding ratios.

\begin{tabular}{|l|c|c|c||c|c|c|}
\hline & \multicolumn{3}{|c|}{ J2E MT systems } & \multicolumn{3}{c|}{ E2J MT systems } \\
\cline { 2 - 7 } & $\mathrm{D}^{3}$ & HPAT & SAT & D $^{3}$ & HPAT & SAT \\
\hline A & 63.7 & 42.5 & 67.2 & 58.4 & 59.6 & 69.8 \\
AB & 72.1 & 63.7 & 74.7 & 72.9 & 75.4 & 81.1 \\
ABC & 78.8 & 79.0 & 82.5 & 83.3 & 86.8 & 88.0 \\
\hline
\end{tabular}

of $1-\alpha \%$.

$$
\left|p_{\text {pro }}-p_{\text {ext }}\right|>t_{(\alpha, 10-1)} * S / \sqrt{10},
$$

where $p_{\text {pro }}$ and $p_{\text {ext }}$, respectively, denote the average performance of the proposed RSCM and the existing RSCM, $t_{(\alpha, 10-1)}$ denotes the upper $\alpha$ point of the Student's t-distribution with $(10-1)$ degrees of freedom, and $S$ denotes the estimated standard deviation of the average difference in performance.

\subsection{Experimental conditions}

\subsubsection{MT systems}

Three English-to-Japanese (E2J) MT systems and three Japanese-to-English (J2E) MT systems of the three types described below were used. Table 2 shows the performances of these MT systems.

$\mathbf{D}^{3}$ (DP-match Driven transDucer) is an example-based MT system using onlinegenerated translation patterns (Doi and Sumita, 2003).

HPAT (Hierarchical Phrase Alignment based Translation) is a pattern-based system using automatically generated syntactic transfer (Imamura et al., 2003).

SAT (Statistical ATR Translator) is an SMT system using a retrieved seed translation as the start point for decoding/translation (Watanabe et al., 2003).

\subsubsection{Test set}

The test set used consists of five hundred and ten pairs of English and Japanese sentences, which

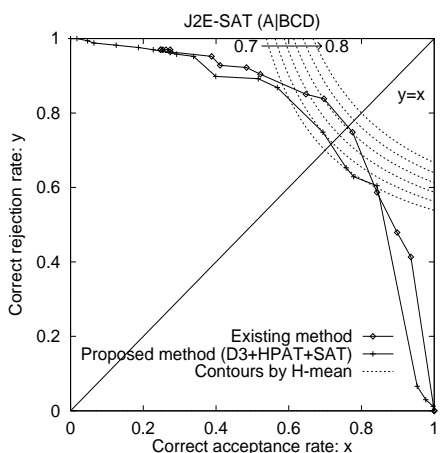

Figure 7: ROC Curves of both RSCMs for J2E-SAT
Table 3: Corpora for training TMs and LMs: Basic Travel Expression Corpus Nos. 1-3 (Takezawa et al., 2002), Travel Reservation Corpus (Takezawa, 1999), and MT-Aided Dialogue Corpus No. 1 (Kikui et al., 2003)

\begin{tabular}{|l|r|r|}
\hline & Japanese & English \\
\hline \# of sentences & \multicolumn{2}{|c|}{449,357} \\
\# of words & $3,471,996$ & $2,978,517$ \\
Vocabulary size & 43,812 & 28,217 \\
Ave. sent. length & 7.7 & 6.6 \\
\hline
\end{tabular}

were randomly selected from the Basic Travel Expression Corpus (BTEC) (Takezawa et al., 2002). BTEC contains a variety of expressions used in a number of situations related to overseas travel.

\subsubsection{Training TMs and LMs}

The corpora used for training TMs and LMs described in Section 3.2 were merged corpora (Table $3)$. The number of trained TMs/LMs was three. The translation models and language models were learned by using GIZA++ (Och and Ney, 2000) and the CMU-Cambridge Toolkit (Clarkson and Rosenfeld, 1997), respectively.

\subsection{Experimental results and discussion}

\subsubsection{ROC Curve}

In order to plot the ROC Curve, the authors conducted the same experiment as shown in Figure 1. That is, in the case where the grade of satisfactory translations is only grade $\mathrm{A}$, each of the proposed and existing RSCMs tried to accept grade A MT outputs and to reject grade B, C, or D MT outputs. Figures 5 to 7 show the ROC Curves for each of the three J2E MT systems (D3, HPAT, and SAT).

The curves with diamond marks, cross marks, triangle marks, and circle marks show the ROC Curves for the existing RSCM, the proposed RSCM by using the mixture of three-best lists from $\mathrm{D}^{3}$, HPAT and SAT, the proposed RSCM by using the mixture of five-best lists from $\mathrm{D}^{3}$ and HPAT, and the existing RSCM with reordering, respectively. In the existing RSCM with reordering, the system's 
Table 4: Ten-fold cross-validated pairwise t-test of H-mean: Each set of three columns corresponds to the experimental results of each of the three MT systems: $\mathrm{D}^{3}$, HPAT, and SAT. Each floating number in the first to third column of each MT system indicates the average performance of the proposed RSCM, the average difference of the performance of the proposed RSCM from that of the existing RSCM, and the t-value of the left-next difference, respectively. The bold floating numbers indicate that the left-next difference is significant at a confidence level of $95 \%$. The floating numbers on the three rows for each MT system, whose row heads are "A $\mid$ BCD", "AB | CD", or "ABC $\mid$ D", correspond to the three types of experiments in which each RSCM tried to accept/reject the MT output assigned one of the grades left/right of "|", respectively.

\begin{tabular}{|c|c|c|c||c|c|c||c|r|r|}
\hline & \multicolumn{3}{|c||}{ E2J-D $^{3}$} & \multicolumn{3}{c||}{ E2J-HPAT } & \multicolumn{3}{c|}{ E2J-SAT } \\
\hline Separating point & Ave. & Diff. & T-val. & Ave. & Diff. & T-val. & Ave. & Diff. & T-val. \\
\hline A $\mid$ BCD & 76.2 & 15.7 & $\mathbf{4 . 4 2 4}$ & 73.2 & 14.1 & $\mathbf{5 . 0 9 9}$ & 65.5 & 0.3 & 0.108 \\
AB | CD & 77.3 & 16.5 & $\mathbf{5 . 1 5 4}$ & 72.6 & 14.3 & $\mathbf{3 . 8 6 5}$ & 66.9 & $2.8 \mathrm{e}-5$ & 0.002 \\
ABC | D & 74.9 & 11.4 & $\mathbf{5 . 9 6 3}$ & 74.7 & 16.6 & $\mathbf{4 . 9 0 6}$ & 73.2 & 5.5 & $\mathbf{2 . 2 8 1}$ \\
\hline \hline \multicolumn{1}{|c|}{} & \multicolumn{3}{|c||}{ J2E-D } & \multicolumn{3}{c||}{ J2E-HPAT } & \multicolumn{3}{c|}{ J2E-SAT } \\
\hline Separating point & Ave. & Diff. & T-val. & Ave. & Diff. & T-val. & Ave. & Diff. & T-val. \\
\hline A $\mid$ BCD & 76.8 & 16.1 & $\mathbf{4 . 9 2 8}$ & 75.5 & 25.8 & $\mathbf{9 . 2 1 8}$ & 70.2 & -3.3 & 1.618 \\
AB | CD & 79.6 & 15.9 & $\mathbf{4 . 9 8 5}$ & 70.8 & 28.9 & $\mathbf{6 . 8 8 5}$ & 66.0 & -5.9 & $\mathbf{2 . 5 4 5}$ \\
ABC | D & 77.7 & 14.4 & $\mathbf{4 . 1 7 7}$ & 71.0 & 22.6 & $\mathbf{4 . 5 9 8}$ & 72.1 & 1.7 & 0.588 \\
\hline
\end{tabular}

Table 5: Ten-fold cross-validated pairwise t-test of Accuracy: The description of this figure is the same as that of Table 4 except that Accuracy is used instead of H-mean.

\begin{tabular}{|c|r|r|r||r|r|r||r|r|r|}
\hline & \multicolumn{3}{|c|}{ E2J-D } & \multicolumn{3}{c||}{ E2J-HPAT } & \multicolumn{3}{c|}{ E2J-SAT } \\
\hline Separating point & Ave. & \multicolumn{1}{|c|}{ Diff. } & \multicolumn{1}{|c|}{ T-val. } & Ave. & \multicolumn{1}{|c|}{ Diff. } & T-val. & Ave. & Diff. & T-val. \\
\hline A $\mid$ BCD & 77.4 & 10.5 & $\mathbf{4 . 3 5 4}$ & 71.1 & 15.4 & $\mathbf{5 . 6 6 7}$ & 76.4 & 1.1 & 1.000 \\
AB | CD & 78.2 & 4.9 & $\mathbf{2 . 9 5 3}$ & 78.2 & 2.5 & $\mathbf{2 . 1 7 6}$ & 81.1 & 0.0 & 0.000 \\
ABC | D & 85.0 & 1.3 & 1.172 & 84.1 & -2.9 & $\mathbf{2 . 1 8 2}$ & 88.0 & 0.0 & 0.000 \\
\hline \hline & \multicolumn{3}{|c|}{ J2E-D } & \multicolumn{3}{c|}{ J2E-HPAT } & \multicolumn{3}{c|}{ J2E-SAT } \\
\hline Separating point & Ave. & \multicolumn{1}{|c|}{ Diff. } & \multicolumn{1}{|c|}{ T-val. } & Ave. & Diff. & T-val. & Ave. & Diff. & T-val. \\
\hline A | BCD & 78.8 & 15.8 & $\mathbf{8 . 2 4 3}$ & 76.2 & 18.2 & $\mathbf{8 . 1 1 8}$ & 76.4 & 3.1 & 1.041 \\
AB | CD & 77.8 & 4.1 & $\mathbf{3 . 2 7 9}$ & 72.7 & 8.8 & $\mathbf{3 . 2 8 8}$ & 77.6 & -1.5 & 0.537 \\
ABC | D & 83.3 & 2.9 & 1.771 & 77.4 & -1.7 & 1.646 & 82.7 & 0.1 & 0.428 \\
\hline
\end{tabular}

original N-best list was sorted by using the average of the product scores from the multiple scoring technique described in Section 3.2, and the existing RSCM with reordering used this sorted system's N-best instead of the system's original N-best. The dotted lines indicate the contours by H-mean from 0.7 to 0.8 . The ideal ROC curve is a square $(0,1),(1,1),(1,0)$; thus, the closer the curve is to a square, the better the performance of the RSCM is.

In Figures 5 and 6, the curves of the proposed RSCM by using the mixture of three-best lists from the three MT systems are much closer to a square than that of the existing RSCM; moreover, the curves of the proposed RSCM by using the mixture of five-best lists from the two MT systems are much closer to a square than that of the existing RSCM. Note that the superiority of the proposed RSCM to the existing RSCM is maintained even in the case where an M-best list from the SMT system was not used. The curves of the existing RSCM with reordering are closer to a square than those of the existing RSCM. Thus the performance of the proposed RSCM on the non-SMT systems, $\mathrm{D}^{3}$ and HPAT, are much better than that of the existing RSCM. The difference between the performance of the proposed and existing RSCMs is due to both resorting the MT outputs and using a mixture of $\mathrm{N}$-best lists.

In Figure 7, the curve of the proposed RSCM is a little closer when CRR is larger than CAR; and the curve of the existing RSCM is a little closer when CAR is larger than CRR. Thus, the performance of the proposed RSCM on the SMT system, SAT, is a little better than that of the existing RSCM in the case where CRR is regarded as important; similarly, the performance of the proposed RSCM on the SMT system is a little worse than that of the existing RSCM in the case where CAR is regarded as important.

\subsubsection{H-mean and Accuracy}

Tables 4 and 5 show the experimental results of tenfold cross-validated pairwise t-tests of the performance of H-mean and Accuracy, respectively.

On the non-SMT systems, Table 4 shows that at every level of translation quality that the user would permit, the H-mean of the proposed RSCM is sig- 
nificantly better than that of the existing RSCM. On the SMT MT system, Table 4 shows that at every permitted level of translation quality, there is no significant difference between the $\mathrm{H}$-mean of the proposed RSCM and that of the existing RSCM except for two cases: "ABC $\mid \mathrm{D}$ " for E2J-SAT and "AB | CD” for J2E- SAT.

Table 5 shows almost the same tendency as Table 4. As for difference, in the case where the translation quality that the user would permit is better than $\mathrm{D}$, there is no significant difference between the Accuracy of the proposed RSCM and that of the existing RSCM except in the one case of "ABC $\mid \mathrm{D}$ " for E2J-HPAT.

As defined in Section 4.1, Accuracy is an evaluation metric whose value is sensitive/inclined to the ratio of the number of satisfactory translations and unsatisfactory translations. H-mean is an evaluation metric whose value is independent/natural to this ratio. We need to use these different evaluation metrics according to the situations encountered. For general purposes, the natural evaluation metric, $\mathrm{H}$ mean, is better. In the case where the test set reflects special situations encountered, Accuracy is useful.

Regardless of whether we encounter any special situation, in most cases on a non-SMT system, the proposed RSCM proved to be significantly better than the existing RSCM. In most cases on an SMT system, the proposed RSCM proved to be as good in performance as the existing RSCM.

This paper reports a case study in which a mixture of N-best lists from multiple MT systems boosted the performance of the RSCM for MT outputs. The authors believe the proposed RSCM will work well only when each of the element MT systems complements the others, but the authors leave the question of the best combination of complementary MT systems open for future study.

\section{Conclusions}

This paper addressed the problem of eliminating unsatisfactory outputs from MT systems. It proposed a method that eliminates unsatisfactory outputs by using an alternative RSCM based on a mixture of N-best lists from multiple MT systems. The authors compared the proposed and existing RSCMs in the framework of an elimination system. When the number of MT outputs both in the N-best list for the existing RSCM and in the mixture of N-best lists for the proposed RSCM is almost the same number, i.e. ten, in most cases, the proposed RSCM proved to work better than the existing RSCM on two nonSMT systems and to work as well as the existing RSCM on an SMT system.
In the future, the authors will conduct the following experiments: (1) investigating how the proposed RSCM works when the size of the M-best lists is increased, and (2) seeing how the proposed RSCM influences the performance of the selection system.

\section{References}

Yasuhiro Akiba, Taro Watanabe, and Eiichiro Sumita. 2002. Using language and translation models to select the best among outputs from multiple MT systems. In Proc. COLING-2002, pages 8-14.

Peter F. Brown, Stephen Della Pietra, Vincent J. Della Pietra, and Robert L. Mercer. 1993. The mathematics of statistical machine translation: Parameter estimation. Computational Linguistics, 19(2):263-311.

Philip Clarkson and Ronald Rosenfeld. 1997. Statistical language modeling using the CMU-Cambridge toolkit. In Proc. EUROSPEECH-1997, pages 2707-2710.

Takao Doi and Eiichiro Sumita. 2003. Input sentence splitting and translating. In Proc. the HLT-NAACL 2003 Workshop on DDMT, pages 104-110.

Kenji Imamura, Eiichiro Sumita, and Yuji Matsumoto. 2003. Feedback cleaning of machine translation rules using automatic evaluation. In Proc. ACL-2003, pages 447-454.

Genichiro Kikui, Eiichiro Sumita, Toshiyuki Takezawa, and Seiichi Yamamoto. 2003. Creating corpora for speechto-speech translation. In Proc. EUROSPEECH-2003, volume 1, pages 381-384.

Vladimir I. Levenshtein. 1966. Binary codes capable of correcting deletions, insertions and reversals. Soviet Physics Doklady, 10(8):707-710.

Tom M. Mitchell. 1997. Machine Learning. The McGraw-Hill Companies Inc., New York, USA.

Sonja Niessen, Franz J. Och, G. Leusch, and Hermann Ney. 2000. An evaluation tool for machine translation: Fast evaluation for machine translation research. In Proc. LREC2000, pages 39-45.

Franz Josef Och and Hermann Ney. 2000. Improved statistical alignment models. In Proc. ACL-2000, pages 440-447.

Kishore A. Papineni, Salim Roukos, Todd Ward, and Wei-Jing Zhu. 2001. Bleu: a method for automatic evaluation of machine translation. In Technical Report RC22176 (W0109022), IBM Research Division, Thomas J. Watson Research Center, Yorktown Heights, NY, pages 257-258.

Eiichiro Sumita, Setsuo Yamada, Kazuhiro Yamamoto, Michael Paul, Hideki Kashioka, Kai Ishikawa, and Satoshi Shirai. 1999. Solutions to problems inherent in spokenlanguage translation: The ATR-MATRIX approach. In Proc. MT Summit VII, pages 229-235.

Toshiyuki Takezawa, Eiichiro Sumita, Fumiaki Sugaya, Hirofumi Yamamoto, and Seiichi Yamamoto. 2002. Toward a broad-coverage bilingual corpus for speech translation of travel conversations in the real world. In Proc. LREC-2002, pages 147-152.

Toshiyuki Takezawa. 1999. Building a bilingual travel conversation database for speech translation research. In Proc. the Oriental COCOSDA Workshop-1999, pages 17-20.

Nicola Ueffing, Klaus Macherey, and Hermann Ney. 2003. Confidence measures for statistical machine translation. In Proc. MT Summit IX, pages 394-401.

Taro Watanabe, Eiichiro Sumita, and Hiroshi G. Okuno. 2003. Chunk-based statistical translation. In Proc. MT Summit IX, pages 410-417. 